\title{
Influence of arm weight support on a robotic assessment of upper limb function
}

\author{
Christoph M. Kanzler ${ }^{1}$, Sofia Martinez Gomez ${ }^{1}$, Mike D. Rinderknecht ${ }^{1}$, Roger Gassert ${ }^{1}$, Olivier Lambercy $^{1}$
}

\begin{abstract}
Quantifying upper limb impairment post-stroke is of essential importance to monitor motor recovery or to evaluate different therapeutic approaches. Instrumented assessments of upper limb function, such as the Virtual Peg Insertion Test (VPIT), often emulate a daily life manipulation activity that requires the subject to actively lift the arm against gravity, which can be challenging for severely impaired patients with arm weakness. With the aim of making the VPIT accessible to patients with severe arm weakness, we conducted a pilot study to analyze the feasability of combining this assessment with an arm weight support (AWS) device in 16 healthy subjects. Subjects performed the VPIT protocol without AWS device and with three different levels of weight support. Usability of combining the VPIT and the AWS device was high in healthy subjects: The VPIT could be successfully completed without collisions with the AWS device, the duration to set up the AWS device was on average $1.5 \mathrm{~min}$, and subjects reported high levels of comfort while experiencing AWS. Metrics representing arm function were mostly not significantly influenced by the presence of the AWS device despite a decrease of $6.2 \%$ in movement smoothness, whereas grasping control was not significantly affected at all. The AWS level did not alter motor performance, even though subjects reported a decrease in perceived arm control with an increased AWS level. The high usability of combining the VPIT with an AWS device might enable the assessment of severely impaired patients in clinical practice. However, the influence of the AWS on outcome measures of the VPIT must be taken into account to make assessment results interpretable in the context of daily life reaching and manipulation situations without AWS.
\end{abstract}

\section{INTRODUCTION}

Assessment of upper limb function after neurological injury, such as stroke, is of primary importance to monitor the motor recovery process during rehabilitation or to evaluate the outcome of new therapeutic approaches [1]. In the last decades, a variety of technology-based assessments (i.e., using robotic platforms or wearable sensors) have been proposed to complement conventional clinical tools, bringing the promises of more objective, sensitive, and standardized scales [?,2-5]. The Virtual Peg Insertion Test (VPIT) is an example of such technology-based assessments, featuring a $3 \mathrm{D}$ pick and place task that requires the user to manipulate objects in a virtual reality environment while actively lifting the arm against gravity, similar to a daily life activity [6].

However, the execution of such a task can be challenging for severely impaired stroke patients due to commonly observed upper limb weakness [7]. The neurophysiological factors underlying this weakness may include pathological

\footnotetext{
${ }^{1}$ Rehabilitation Engineering Laboratory, Department of Health Sciences and Technology, ETH Zurich, Switzerland. christoph.kanzler@hest.ethz.ch
}

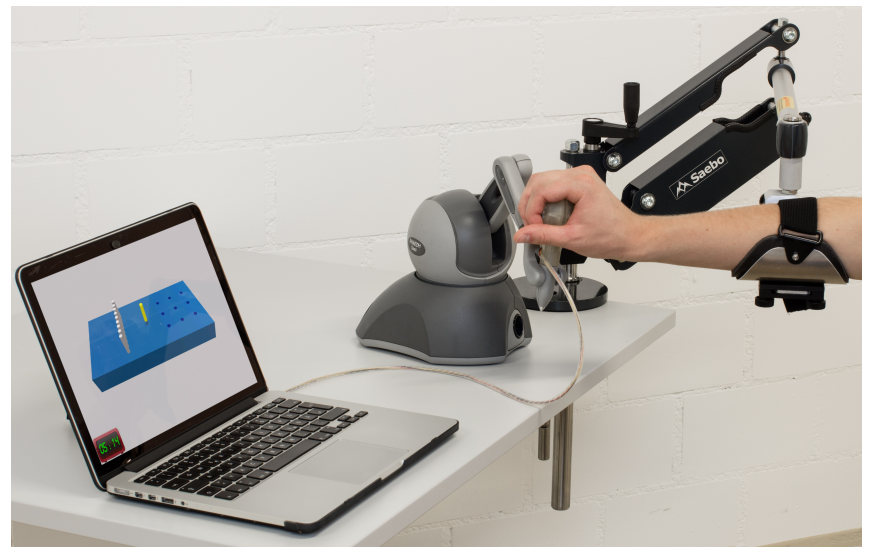

Fig. 1. The Virtual Peg Insertion Test (VPIT) for assessing upper limb function with the selected passive arm support device (SaeboMAS mini).

muscle coactivation patterns that lead to an abnormal coupling of shoulder abduction with elbow flexion torques [8], as well as peripheral and central fatigue due to muscle fiber rearrangement [9] and inhibitory neural mechanisms, respectively [10]. Indeed, in a previous study with the VPIT, $65 \%$ of the stroke patients that were assessed on their mostaffected limb could not complete the test without arm weight support (AWS) from their less-affected limb [11]. However, this way of providing AWS limits the repeatability of the assessment and its comparison within and across subjects, as the AWS level cannot be controlled objectively and may vary within and between participants.

In order to provide AWS in a reproducible and systematic way, active and passive devices have been used for poststroke neurorehabilitation and were able to increase arm workspace during and after training with an AWS [12-14], and to reduce the coupling of the shoulder-elbow synergy while being supported $[13,15]$. For most technology-based assessments of upper limb motor function other than the VPIT, AWS is directly provided by the assessment platform in several paradigms, as participants are often performing movements while being supported by the device itself (e.g., KINARM [16], MIT-MANUS [17], ArmeoSpring (Hocoma AG, Switzerland)). The provided AWS might augment motor function, which limits the transferability of assessment outcomes to daily life reaching situations and thereby creates the necessity to model the influence of AWS on motor performance. However, this influence can only be quantified if the user performs the assessment with and without the AWS, which is not possible in case the support is provided by the assessment platform itself.

We analyze the feasibility of combining the VPIT with 
a passive, external AWS device in healthy subjects, which could open new possibilities for this technology-based assessment, making it accessible for the evaluation of severely impaired neurological patients. Our aims were to (i) select a passive AWS device suitable for the VPIT in terms of usability and potential for application in clinical practice, (ii) evaluate the feasibility of combining the VPIT with the chosen AWS, and (iii) measure the influence of the AWS on kinematics and kinetics recorded with the VPIT. We hypothesized that in healthy subjects, AWS would have a slight, negative influence on upper limb motor performance due to the obstructive nature of the AWS device for healthy subjects $[18,19]$.

\section{METHODS}

\section{A. Virtual Peg Insertion Test}

The VPIT features a 3D pick and place task rendered through a haptic device (Touch, 3D Systems, CA, USA) and a virtual reality environment (Fig. 1). The haptic device has a workspace of approximately $16 \times 12 \times 7 \mathrm{~cm}$ (width, height, depth) and measures 3D movement kinematics of the endeffector. Additionally, a custom-made handle with three single-axis force sensors (CentoNewton40, EPFL, Switzerland) enables the recording of grasping forces [6]. The task requires the subject to select and lift nine pegs by applying adequate grasping force (i.e., above a $2 \mathrm{~N}$ threshold) and transport them into corresponding holes. For a more detailed description of the VPIT as well as examples of raw position and force profiles, please refer to earlier work [6] and previous studies with stroke, multiple sclerosis and ataxic patients [11, 20-22].

\section{B. Requirements for an AWS device}

The objective was to select a commercially available, passive (i.e., weight compensation is achieved through a spring mechanism rather than motors) and transparent (i.e., low friction and inertia of the device and thereby small interaction forces between device and user) AWS device that can support the arm throughout the 3D space for implementation with the VPIT. A passive system was preferred over an actuated solution, as the latter would be less cost-effective and portable than a passive implementation. The AWS device should be able to provide adjustable support up to a maximum level of approximately $50 \mathrm{~N}$, such that persons with up to $100 \mathrm{~kg}$ body weight could benefit from the AWS (assuming the arm weight being $5 \%$ of the body weight [23]). Additionally, the device should be portable and table-mounted to maintain the high transportability of the VPIT and thereby a simple deployment of both tools into clinical environments. Most importantly, the AWS device should have a workspace that covers the functional workspace of the VPIT such that participants can successfully complete the assessment with AWS device without interfering with the haptic device. Finally, usability in terms of comfort for the user, low effort and time for setting up and adjusting the AWS, as well as the possibility to use the system for both left and right arm should be considered.

\section{Selection of an AWS for the VPIT}

The Armeo Boom (Hocoma, Switzerland), Armon Edero (Armon products B.V., Netherlands), Armon Pura, SaeboMAS (Saebo, NC, USA), SaeboMAS mini (Saebo, NC, USA) and the WREX (JAECO Orthopedic, AR, USA), which are typical commercial AWS devices used for rehabilitation or assistance of patients with arm impairments $[14,18,24]$, were considered as possible AWS devices for the VPIT. The Armeo Boom and the SaeboMAS were excluded as they are not easily transportable. The Armon Edero and the WREX were discarded because they cannot be easily used with both the left and right arm, whereas the Armon Pura was not considered as it needs to be attached to a certain type of chair, which can limit clinical applicability. Instead, the SaeboMAS mini (Fig. 1) was chosen as it combines the advantage of being a passive, table-mounted, springbased AWS that can easily be transported and adjusted to the user. In more detail, the SaeboMAS mini has a weight of $4.9 \mathrm{~kg}$ and a height and length of $53.3 \mathrm{~cm}$ and $40.6 \mathrm{~cm}$ in closed state, respectively. The device can provide up to $38.2 \mathrm{~N}$ of AWS throughout the 3D space using a springbased parallelogram structure, which can be extended to a length of up to $74.9 \mathrm{~cm}$ in horizontal direction. The tension of the spring system and thereby the level of support can be mechanically adjusted using a winch. The usability and simplicity of the SaeboMAS mini make it a suitable solution for integration with the VPIT, even though the maximal level of AWS did not fulfill the defined requirements.

\section{Participants}

Sixteen healthy subjects $(25.1 \pm 1.6 \mathrm{yrs}, 67.8 \pm 13.6 \mathrm{~kg}$, 8 female, all self-reported right hand dominant; mean \pm standard deviation) were recruited at ETH Zurich. Before participation, all subjects gave written informed consent and all experimental procedures were approved by the ethics committee of ETH Zurich (2017-N-35).

\section{E. Experimental protocol}

Participants were seated in a chair in front of a heightadjustable table. The AWS device was mounted on the side edge of the table at $20 \mathrm{~cm}$ distance of the table's front edge. The haptic device of the VPIT was placed with a distance of $20 \mathrm{~cm}$ to the front edge of the table and a distance of $30 \mathrm{~cm}$ to the AWS. The height of the table was adjusted such that the participant had a shoulder abduction angle of $45^{\circ}$, a shoulder flexion angle of $10^{\circ}$ and an elbow flexion angle of $90^{\circ}$ in initial position (i.e., hand on the handle that is resting in the haptic device). The forearm of the participant was placed in the support base of the AWS device with a distance to the elbow joint of approximately one third of the lower arm length (Figure 1). The amount of pretension needed to achieve an AWS level of $100 \%$ (relative to the arm weight) was estimated experimentally for each participant. In detail, the AWS was attached to the subject with minimal amount of pretension and with the subject being instructed to remain passive. The amount of pretension was then increased incrementally until the parallelogram structure of the support 


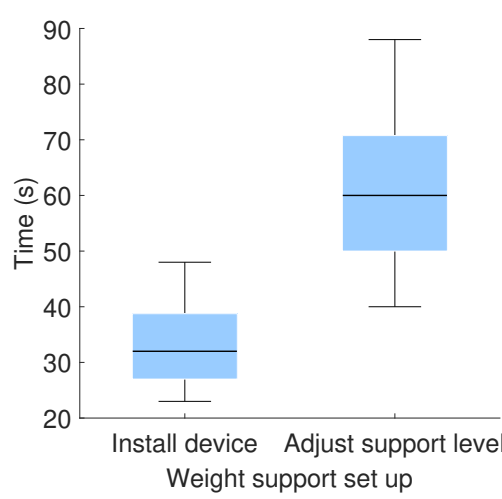

(a)

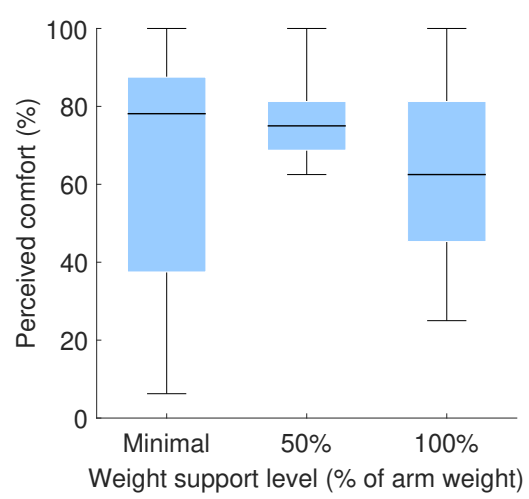

(b)

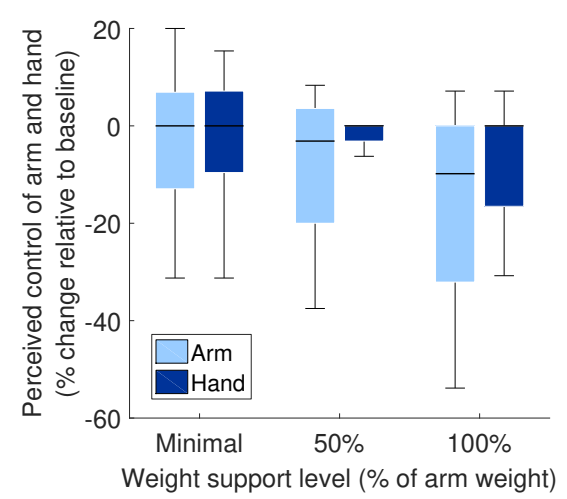

(c)

Fig. 2. Usability (a), comfort (b) and perceived upper limb control (c) when using an arm weight support with the Virtual Peg Insertion Test (VPIT).

device was horizontal (i.e., angle between parallelogram and vertical bar of $90^{\circ}$ ).

Experiments were performed under four conditions and on two different days, with 2-5 days break in between to limit learning effects on the VPIT task. On the first day, subjects performed the VPIT without the AWS device as well as with $100 \%$ AWS. On the last day, participants completed the VPIT with 50\% AWS as well as with minimal AWS (i.e., AWS device with minimal pretension attached to the arm). Each of the four conditions consisted of a set up period (i.e., mounting the AWS and adjusting the AWS level), an instruction period (i.e., explanation and familiarization with the task), five repetitions of the VPIT (i.e., inserting all pegs five times), and a questionnaire. Subjects were pseudorandomly assigned to four experimental groups such that the order of conditions within each day was randomized.

The questionnaire included 10 usability-related questions that were inspired from the NASA Task Load Index [25] and had to be answered on a visual analog scale (VAS). In this work, we focus on the outcome of the questions "how comfortable was the arm weight compensation?", "did you feel in control of your arm" and "did you feel in control of your hand" with the VAS ranging from "not at all" to "very much", which was transformed post-hoc into a scale ranging from $0 \%$ to $100 \%$. The questions regarding the perceived control of arm and hand were additionally normalized to the baseline condition (i.e., VPIT without the AWS device).

\section{F. Data Analysis}

1) Usability of combining the VPIT and the AWS device: Usability was assessed using the success rate (i.e., if all pegs could be inserted) in the VPIT while experiencing AWS and the perceived comfort quantified through the questionnaire. Clinical usability was evaluated by the time required to install the AWS on the table (from the device in closed position on the table to successful attachment) and the time to adjust the AWS level (from when the experimenter initiated the adjustment of the AWS level from minimum pretension until an AWS level of $100 \%$ was reached for each subject). Box plots were used to display median, interquartile ranges (IQR) and the minimum/maximum value still within $1.5 \mathrm{IQR}$ of the lower/upper quartile for the time to set up the AWS and the perceived comfort. Tests for statistical significance related to the questionnaire were performed using Friedman tests (significance level of 5\%).

2) Influence of AWS on upper limb function: Results for the questions regarding perceived control of arm and hand were visualized as box plots.

Additionally, kinematic and kinetic metrics were extracted from position and force traces measured by the VPIT to enable an assessment of different aspects of motor control (refer to [6] for details). In short, overall motor performance was described by the total time to complete a repetition, which was defined as the time between lifting the first peg until the insertion of the last peg [21]. Movement smoothness is an indicator of movement coordination and was quantified using the normalized, dimensionless jerk [26] and the spectral arc length [27], which were calculated during the gross movement phases of the task. The efficiency of movements is constrained through the speed-accuracy tradeoff, which was measured using the throughput based on the modified version of Fitt's law, which relates both gross movement length and duration to the 3D endpoint error [28]. Grasp force control was analyzed using the maximally applied force, the maximum rate of force development after the grasp of a peg and before the maximal force level was reached, and the maximum rate of force release during the insertion of a peg.

Linear mixed effects models (LME) were generated for each metric to model the influence of the presence of the AWS device and the AWS level, but also confounding factors such as possible learning effects across experimental sessions. Metrics were normalized with respect to the experimental condition without the AWS device (i.e., subtraction and division by absolute baseline value for each subject). Each LME contained fixed effects for intercept, presence of the AWS device (with/without device), AWS level (in percent of arm weight), number of experimental session (ordinal entry ranging from 1 to 4 ) to represent the influence of learning as well as subject-specific intercepts. The contribution of fixed effects to model quality was evaluated using simulated likelihood ratio tests $(n=1000,5 \%$ significance level) based on the complete LME and a reduced model that did not contain the analyzed fixed effect [29]. Estimates and standard errors (SE) of the coefficients as well as $p$-values were 


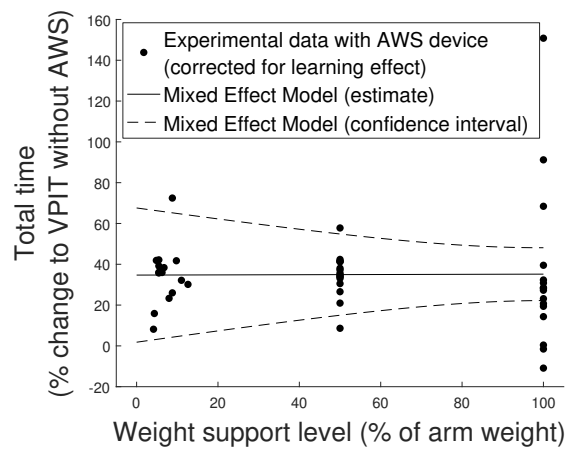

(a)

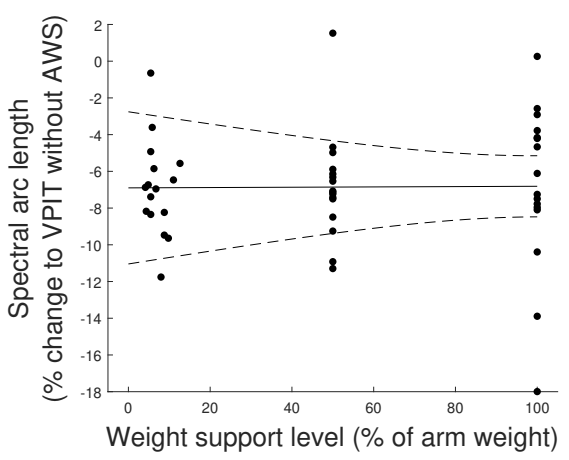

(b)

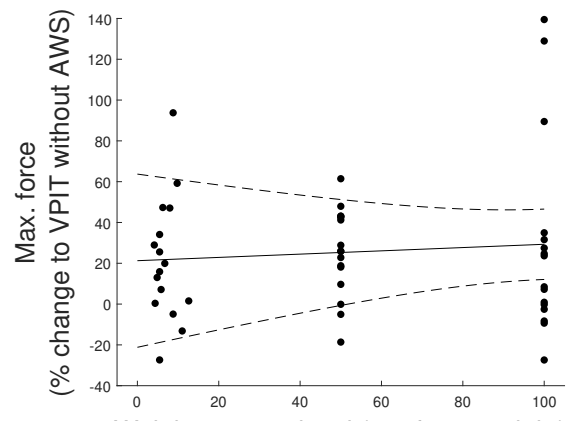

Weight support level (\% of arm weight)

(c)

Fig. 3. Influence of the arm weight support device (one factor contributing to intercept of model) and the support level (slope of model) on overall motor control (total time), arm control (spectral arc length and thereby smoothness) and one of the metrics representing grasping control (max. force) as recorded with the Virtual Peg Insertion Test (VPIT). The mixed effects models were used to compensate for learning effects in the experimental data.

reported for fixed effects. The standard deviation (SD) was calculated for random effects. Quality of fit was evaluated using the coefficient of determination $\left(R^{2}\right)$.

The influence of the AWS level on overall motor control (higher values of the total time indicate reduced control), arm control (lower values of the spectral arc length indicate lower smoothness) and grasping control (higher values of the maximum force indicate less efficient force control) was visualized based on the LMEs (estimates and 95\% confidence intervals) and the experimental data points, which were corrected for learning effects using the generated LMEs.

\section{RESULTS}

\section{A. Usability of combining the VPIT and the AWS device}

The VPIT was successfully completed while experiencing AWS during 240 out of 240 repetitions without any collisions between VPIT and the AWS device. The time to install and adjust the AWS is depicted in Fig. 2 (a) and was $32.0 \mathrm{~s}$ $[27.0 \mathrm{~s}, 39.0 \mathrm{~s}]$ and $60 \mathrm{~s}[50.0 \mathrm{~s}, 71.5 \mathrm{~s}]$, respectively (reported as median [lower quartile, upper quartile]). The perceived comfort of the AWS device for a support level of $0 \%, 50 \%$ and $100 \%$ is displayed in Fig. 2 (b) and was $78.1 \%$ [37.5\%, $87.5 \%$ ], $75.0 \%$ [68.8\%, 81.3\%] and $62.5 \%$ [45.3\%, $81.3 \%$ ], respectively, but the difference between conditions was not statistically significant $(p=0.98)$.

\section{B. Influence of AWS on upper limb motor function}

The change (relative to baseline) in perceived arm control was $0.0 \%[-12.9 \%, 6.9 \%],-3.1 \%$ [-20.0\%, 3.6\%] and $-9.8 \%$ $[-32.1 \%, 0.0 \%]$ for an AWS level of $0 \%, 50 \%$ and $100 \%$, respectively, whereas it was $0.0 \%[-9.6 \%, 7.1 \%], 0.0 \%[-3.1 \%$, $0.0 \%]$, and $0.0 \%$ [-16.5\%, $0.0 \%]$ for the perceived control of the hand, respectively (Fig. 2 (c)). The changes for arm and hand control were not statistically significant between conditions with $p$-values of 0.07 and 0.68 , respectively.

An overview of the LMEs is given in Table 1. The median coefficient of determination was 0.48 [0.40, 0.62] across models. Using the AWS device lead to a statistically significant decrease in smoothness (spectral arc length decreased by $6.2 \%$ ) relative to baseline (Fig. 3 (b)). The AWS level did not significantly change motor performance according to the analyzed metrics (examples in Fig. 3 a-c). The total time (overall motor control), normalized jerk (movement smoothness) and throughput (movement efficiency) metrics were statistically significantly improved by $49.3 \%, 39.7 \%$ and $27.2 \%$ through learning effects across experimental sessions, respectively.

\section{DISCUSSION}

The objective of this work was to evaluate the feasibility of combining the VPIT with an AWS device by quantifying usability of the combined system and the effect of the AWS device and support level on the outcome measures of the VPIT in young healthy subjects.

\section{A. Usability of combining the VPIT and the AWS device}

The selected AWS device showed high usability for the VPIT. All subjects were able to successfully complete the test while experiencing AWS and the portability, ease of use, and short duration to set up the device makes it clinically applicable. Additionally, subjects reported a high level of perceived comfort, even though a trend towards slightly less comfort with an increasing level of AWS was observed. This might be explained by the fact that healthy subjects do not need support to successfully complete their movements. AWS can therefore be perceived as an obstructive factor with a disturbing influence that scales with the level of AWS.

One shortcoming of the selected support device is the necessity to adjust the AWS level manually to the unknown arm weight of each patient without a scale that indicates the absolute level of provided force. This could potentially lead to low reliability for the adjustment of the AWS level, as the accuracy and precision of the adjustment is dependent on the experience of the experimenter. Another shortcoming is the maximal level of AWS provided by the support device that did not fulfill the predefined requirements and therefore limits the application to subjects with a body weight equal or below approximately $80 \mathrm{~kg}$.

Despite these shortcomings, the usability is promising for an application of the VPIT and the AWS device in patients with severe arm weakness. Especially, the high comfort, portability, and short duration to set up the device could enable an integration into clinical routine. 
TABLE I

INFLUENCE OF THE ARM WEIGHT SUPPORT (AWS) AND LEARNING EFFECTS ON OUTCOME MEASURES OF THE VIRTUAL PEG INSERTION TEST (VPIT) BASED ON MIXED EFFECTS MODEL ANALYSIS. P-VALUES DESCRIBE WHETHER THE EFFECT STATISTICALLY SIGNIFICANCTLY IMPROVED MODEL QUALITY. METRICS WERE NORMALIZED RELATIVE TO THE EXPERIMENTAL CONDITION WITHOUT AWS DEVICE.

\begin{tabular}{|c|c|c|c|c|c|c|c|c|c|}
\hline \multirow[t]{3}{*}{ Metrics } & \multicolumn{7}{|c|}{ Fixed effects } & \multirow{3}{*}{$\begin{array}{c}\text { Random effects } \\
\text { (Intercept) } \\
\text { SD }\end{array}$} & \multirow[t]{3}{*}{$\mathbf{R}^{2}$} \\
\hline & \multirow{2}{*}{$\begin{array}{c}\text { (Intercept) } \\
\text { Estimate (SE) }\end{array}$} & \multicolumn{2}{|c|}{ Presence of AWS device ${ }^{1}$} & \multicolumn{2}{|c|}{ AWS level $^{2}$} & \multicolumn{2}{|c|}{ Learning $^{3}$} & & \\
\hline & & Estimate (SE) & $p$-value & Estimate (SE) & $p$-value & Estimate (SE) & $p$-value & & \\
\hline \multicolumn{10}{|c|}{ Overall motor control } \\
\hline Total time & $15.58(6.47)$ & $19.13(16.45)$ & 0.276 & $0.0(0.15)$ & 0.976 & $-49.27(10.85)$ & $<0.001$ & 12.73 & 0.63 \\
\hline \multicolumn{10}{|c|}{ Movement smoothness } \\
\hline Normalized jerk & $12.01(8.08)$ & 40.93 (19.52) & 0.055 & $-0.18(0.18)$ & 0.34 & $-39.65(12.87)$ & 0.005 & 18.17 & 0.48 \\
\hline Spectral arc length & $-0.67(0.83)$ & $-6.23(2.06)$ & 0.006 & $0.0(0.02)$ & 0.965 & $2.54(1.36)$ & 0.201 & 1.75 & 0.59 \\
\hline \multicolumn{10}{|c|}{ Movement efficiency } \\
\hline Throughput & $-6.81(3.69)$ & $-11.3(8.32)$ & 0.211 & $0.02(0.08)$ & 0.819 & $27.24(5.49)$ & $<0.001$ & 9.36 & 0.74 \\
\hline \multicolumn{10}{|l|}{ Force control } \\
\hline Max. force & $11.68(8.62)$ & $9.58(21.1)$ & 0.672 & $0.08(0.19)$ & 0.703 & $-19.6(13.91)$ & 0.076 & 18.77 & 0.4 \\
\hline Force buildup rate & $7.04(10.26)$ & $4.25(24.73)$ & 0.874 & $0.1(0.23)$ & 0.675 & $-4.22(16.31)$ & 0.472 & 23.15 & 0.38 \\
\hline Force falloff rate & $8.64(9.81)$ & $24.81(23.65)$ & 0.325 & $-0.06(0.22)$ & 0.798 & $-15.41(15.59)$ & 0.377 & 22.16 & 0.4 \\
\hline
\end{tabular}

SE: standard error; SD: standard deviation; ${ }^{1}$ baseline: false; ${ }^{2}$ decimal $\in[0,100]$ (\% of arm weight); ${ }^{3}$ ordinal $\in[1,4]$; averaged estimate and SE reported.

\section{B. Influence of AWS on upper limb function}

The presence of the AWS device did not have major effects on upper limb function with only a minor, statistically significant reduction in smoothness of $6.2 \%$ according to the spectral arc length metric. Additionally. there was a considerable but not statistically significant reduction in performance with the presence of the AWS device according to the total time $(19.1 \%)$, normalized jerk $(40.9 \%)$ and force falloff rate $(24.9 \%)$ metrics, whereas the AWS level did not significantly influence motor performance. These findings confirm our hypothesis and are partly in line with the work of Coscia et al. [18], who showed only minor changes in motor performance, but also reduced movement smoothness with an increased AWS level. Pirondini et al. also reported decreased movement smoothness but additionally improved accuracy when comparing reaching with an exoskeleton to natural reaching movements, which was attributed to improved attention to the task due to the unusual situation of wearing an exoskeleton [30].

The minor negative influence of the AWS device on movement smoothness and the independence of the metrics to the AWS level partly contradicts the results of the questionnaires, which instead indicated a trend towards a decrease in perceived arm but not hand control with an increased AWS level that was, however, not statistically significant.

The slightly reduced motor performance in healthy subjects might stem from reduced proximal muscle activity that leads to a change in sensory feedback $[18,19]$. In general, goal-directed movements are executed based on neural, parametric models that translate motor commands into wellcoordinated movements [31]. The parameters of these models are learned and adapted to the body characteristics of each individual. Modifying the input to these highly optimized models, in this case through the change of sensory feedback due to a reduction in proximal muscle activity, leads to suboptimal outputs of the model, thereby altered movements and ultimately reduced motor performance. However, we would expect the reduced proximal muscle activity to be dependent on the AWS level, which should therefore systematically influence motor performance. The findings from this study thereby contradict this hypothesis. Interaction forces between the user and AWS device due to friction or inertia of the device could potentially also alter motor performance. However, we would expect this effect to lead to a systematic change across kinematic metrics when using the AWS device, which was not reflected in our experimental data and might instead suggest that the AWS device has negligible friction and inertia. Another contributing factor to the slightly altered motor performance could also be behavioural changes (e.g., altered attention) associated with the usage of the AWS device, which should, however, also lead to systematic effects across all aspects of arm control.

A limitation of this feasibility study is the absence of experiments with neurologically impaired subjects. It could be expected that AWS would have a different influence in a patient population with severe motor impairment, as the reduction of proximal muscle activity through AWS could help to overcome synergistic movement patterns and fatigue effects, as shown in previous work $[12,15,32]$. Specifically, we would expect increased reaching work area [12] and reduced coupling of the shoulder-elbow synergy [15], which could also lead to increased grasping control [32], thereby potentially enabling the VPIT for severely impaired patients that could not perform the assessment without AWS. However, the augmentation effect of the AWS would change the interpretation of the outcome measures of the VPIT, which is supposed to capture activity-oriented motor impairments that might be transferable to daily life reaching and manipulation situations. This would create the necessity to model the influence of the AWS on outcome measures to preserve the possibility to draw conclusions about activity-oriented motor skills. Even though the AWS would augment upper limb motor function in patients, we expect the influence to be systematic and reproducible, which could enable valid intra- 
and inter-subject comparisons and high test-retest reliability.

\section{CONCLUSION}

The evaluated AWS device demonstrated high usability for the VPIT and had a minor influence on upper limb motor performance when tested in healthy subjects. This paves the way for applications in patients with severe arm weakness.

\section{ACKNOWLEDGEMENTS}

This project has received funding from the European Union's Horizon 2020 research and innovation programme under grant agreement No. 688857 (SoftPro). The authors would like to thank Hankamp Rehab for providing the SaeboMAS mini used in this study.

\section{REFERENCES}

[1] O. Lambercy, L. Lünenburger, R. Gassert, and M. Bolliger, "Robotic and wearable sensor technologies for measurements/clinical assessments," in Neurorehabilitation technology, 2nd ed. Springer, 2016, pp. $443-456$.

[2] A. M. Coderre, Amr Abou Zeid, S. P. Dukelow, M. J. Demmer, K. D. Moore, M. J. Demers, H. Bretzke, T. M. Herter, J. I. Glasgow, K. E. Norman, S. D. Bagg, and S. H. Scott, "Assessment of Upper-Limb Sensorimotor Function of Subacute Stroke Patients Using Visually Guided Reaching," Neurorehabil Neural Repair, vol. 24, no. 6, pp. 528-541, 2010.

[3] E. Otaka, Y. Otaka, S. Kasuga, A. Nishimoto, K. Yamazaki, M. Kawakami, J. Ushiba, and M. Liu, "Clinical usefulness and validity of robotic measures of reaching movement in hemiparetic stroke patients." J Neuroeng Rehabil, vol. 12, no. 1, p. 66, 2015.

[4] H. I. Krebs, M. Krams, D. K. Agrafiotis, A. Di Bernardo, J. C. Chavez, G. S. Littman, E. Yang, G. Byttebier, L. Dipietro, A. Rykman, K. McArthur, K. Hajjar, K. R. Lees, and B. T. Volpe, "Robotic measurement of arm movements after stroke establishes biomarkers of motor recovery," Stroke, vol. 45, no. 1, pp. 200-204, 2014.

[5] A. Panarese, E. Pirondini, P. Tropea, B. Cesqui, F. Posteraro, and S. Micera, "Model-based variables for the kinematic assessment of upper-extremity impairments in post-stroke patients." J Neuroeng Rehabil, vol. 13, no. 1, p. 81, 2016.

[6] M. Fluet, O. Lambercy, and R. Gassert, "Upper limb assessment using a Virtual Peg Insertion Test," in Proceedings of the 11th IEEE ICORR, 2011, pp. 1-6.

[7] E. S. Lawrence, C. Coshall, R. Dundas, J. Stewart, a. G. Rudd, R. Howard, and C. D. Wolfe, "Estimates of the prevalence of acute stroke impairments and disability in a multiethnic population." Stroke, vol. 32, no. 6, pp. 1279-1284, 2001.

[8] J. P. A. Dewald and R. F. Beer, "Abnormal joint torque patterns in the paretic upper limb of subjects with hemiparesis," Muscle \& Nerve, vol. 24, no. 2, pp. 273-283, 2001.

[9] R. Dattola, P. Girlanda, G. Vita, M. Santoro, M. Roberto, A. Toscano, C. Venuto, A. Baradello, and C. Messina, "Muscle rearrangement in patients with hemiparesis after stroke: An electrophysiological and morphological study," Eur Neurol, vol. 33, no. 2, pp. 109-114, 1993.

[10] S. Knorr, T. D. Ivanova, T. J. Doherty, J. A. Campbell, and S. J. Garland, "The origins of neuromuscular fatigue post-stroke," Exp Brain Res, vol. 214, no. 2, pp. 303-315, 2011.

[11] B. C. Tobler-Ammann, E. D. de Bruin, M.-C. Fluet, O. Lambercy, R. A. de Bie, and R. H. Knols, "Concurrent validity and test-retest reliability of the Virtual Peg Insertion Test to quantify upper limb function in patients with chronic stroke." J Neuroeng Rehabil, vol. 13, no. 1 , p. $8,2016$.

[12] M. D. Ellis, T. Sukal, T. Demott, and J. P. A. Dewald, "Augmenting clinical evaluation of hemiparetic arm movement with a laboratorybased quantitative measurement of kinematics as a function of limb loading," Neurorehabil Neural Repair, vol. 22, no. 4, pp. 321-329, 2008.

[13] T. Krabben, G. B. Prange, B. I. Molier, A. H. Stienen, M. J. Jannink, J. H. Buurke, and J. S. Rietman, "Influence of gravity compensation training on synergistic movement patterns of the upper extremity after stroke, a pilot study," J Neuroeng Rehabil, vol. 9, no. 1, pp. 1-12, 2012.
[14] G. B. Prange, A. I. Kottink, J. H. Buurke, M. M. Eckhardt, B. J. Van Keulen-Rouweler, G. M. Ribbers, and J. S. Rietman, "The effect of arm support combined with rehabilitation games on upperextremity function in subacute stroke: A randomized controlled trial," Neurorehabil Neural Repair, vol. 29, no. 2, pp. 174-182, 2015.

[15] R. F. Beer, M. D. Ellis, B. G. Holubar, and J. P. Dewald, "Impact of gravity loading on post-stroke reaching and its relationship to weakness," Muscle \& Nerve, vol. 36, no. 2, pp. 242-250, aug 2007.

[16] S. H. Scott, "Apparatus for measuring and perturbing shoulder and elbow joint positions and torques during reaching," Journal of Neuroscience Methods, vol. 89, no. 2, pp. 119-127, 1999.

[17] H. Krebs, N. Hogan, M. L. Aisen, and B. T. Volpe, "Robot-aided neurorehabilitation," IEEE Trans Rehabil Eng, vol. 6, no. 1, pp. 7587, 1998.

[18] M. Coscia, V. C. Cheung, P. Tropea, A. Koenig, V. Monaco, C. Bennis, S. Micera, and P. Bonato, "The effect of arm weight support on upper limb muscle synergies during reaching movements," J Neuroeng Rehabil, vol. 11, no. 1, p. 22, 2014.

[19] K. D. Runnalls, G. Anson, and W. D. Byblow, "Partial weight support of the arm affects corticomotor selectivity of biceps brachii," J Neuroeng Rehabil, vol. 12, no. 1, pp. 1-10, 2015.

[20] O. Lambercy, M.-C. Fluet, I. Lamers, L. Kerkhofs, P. Feys, and R. Gassert, "Assessment of upper limb motor function in patients with multiple sclerosis using the Virtual Peg Insertion Test: A pilot study," in Proceedings of the 13th IEEE ICORR, 2013, pp. 1-6.

[21] C. Gagnon, C. Lavoie, I. Lessard, J. Mathieu, B. Brais, J. P. Bouchard, M. C. Fluet, R. Gassert, and O. Lambercy, "The Virtual Peg Insertion Test as an assessment of upper limb coordination in ARSACS patients: A pilot study," J Neurol Sci, vol. 347, no. 1-2, pp. 341-344, 2014.

[22] P. Hofmann, J. P. Held, R. Gassert, and O. Lambercy, "Assessment of movement patterns in stroke patients: A case study with the virtual peg insertion test," in Proceedings of i-CREATe, 2016, pp. 1-4.

[23] C. E. Clauser, J. T. McConville, and J. W. Young, "Weight, Volume, and Center of Mass of Segments of the Human Body," National Technical Information Service, pp. 1-112, 1969.

[24] E. Hallewell, T. Excell, K. Meadmore, C. Freeman, M. Kutlu, A.M. Hughes, and J. Burridge, "Goal-orientated functional rehabilitation using electrical stimulation and iterative learning control for motor recovery in the upper extremity post-stroke." in 18th Annual Conference of the International Functional Electrical Stimulation Society, 2013.

[25] S. G. Hart and L. E. Staveland, "Development of NASA-TLX (Task Load Index): Results of Empirical and Theoretical Research," in Human Mental Workload, P. Hancock and N. Meshkati, Eds. Elsevier, 1988, pp. 139-183.

[26] N. Hogan and D. Sternad, "Sensitivity of Smoothness Measures to Movement Duration, Amplitude, and Arrests," J Mot Behav, vol. 41, no. 6, pp. 529-534, nov 2009.

[27] S. Balasubramanian, A. Melendez-Calderon, and E. Burdet, "A robust and sensitive metric for quantifying movement smoothness," IEEE Trans Biomed Eng, vol. 59, no. 8, pp. 2126-2136, 2012.

[28] P. M. Fitts and J. R. Peterson, "Information capacity of discrete motor responses." J Exp Psychol, vol. 67, no. 2, pp. 103-112, 1964.

[29] M. D. Rinderknecht, O. Lambercy, V. Raible, J. Liepert, and R. Gassert, "Age-based model for metacarpophalangeal joint proprioception in elderly," Clin Interv Aging, vol. 12, pp. 635-643, 2017.

[30] E. Pirondini, M. Coscia, S. Marcheschi, G. Roas, F. Salsedo, A. Frisoli, M. Bergamasco, and S. Micera, "Evaluation of the effects of the Arm Light Exoskeleton on movement execution and muscle activities: A pilot study on healthy subjects," J Neuroeng Rehabil, vol. 13, no. 1, pp. 1-21, 2016.

[31] J. W. Krakauer, "Motor learning: its relevance to stroke recovery and neurorehabilitation." Curr Opin Neurol, vol. 19, no. 1, pp. 84-90, 2006.

[32] Y. Lan, J. Yao, and J. P. Dewald, "The Impact of Shoulder Abduction Loading on Volitional Hand Opening and Grasping in Chronic Hemiparetic Stroke," Neurorehabil Neural Repair, vol. 31, no. 6, pp. 521-529, 2017. 